\title{
Pre-service teachers' experience with Khan Academy in introductory physics
}

\author{
Christine Lindstrøm and James Gray \\ Faculty of Education and International Studies, \\ Oslo and Akershus University College of Applied Sciences, \\ $\mathrm{PB} 4 \mathrm{St}$. Olavs plass, N-0130 Oslo, Norway
}

(Dated: September 29, 2017)

\begin{abstract}
As a novel approach to the longstanding issue of how to help all students effectively learn and utilize math in physics, we integrated the free online mathematics learning system Khan Academy (KA) into introductory physics for pre-service teachers. Two focus group interviews $(N=11)$ were conducted: one group had shown significant improvement on a math diagnostic test and the other had not. Thematic analysis revealed that the way KA was integrated into the course fostered internalization of the motivation for using KA; students displayed variability in depth of cognitive engagement in response to the feedback mechanism in $\mathrm{KA}$; and $\mathrm{KA}$ helped students get into a 'mathematical mindset,' which enabled some students to forge deeper connections between math and physics. We also suggest fruitful avenues for further research on this longstanding issue in PER.

Keywords: Physics education research; mathematization; preservice teachers; undergraduate; educational computer software
\end{abstract}

\section{BACKGROUND}

Redish and Kuo [1] recently pointed out that "despite much research ... the problem of how to effectively include math in physics in a way that reaches most students remains unsolved" (p. 561). In their article, they explore many of the interacting elements that contribute to the complex whole involved in using math in physics, making a convincing case for why this is such a challenge for the PER community. With the support of the Norwegian Centre for Research, Innovation and Coordination of Mathematics Teaching (MatRIC), we ran a pilot study that incorporated the online learning system Khan Academy (KA) into introductory physics, which provided a practical and productive test-bed. We report on emerging insights from this project that indicate directions for addressing the difficult problem above.

$\mathrm{KA}$ is a free online learning system. It spans a number of subject areas, but the math component is the most well-developed both in terms of quantity and integration of the various features making up the system. Hundreds of topics - from counting to calculus - are systematically organized. To complete a topic, the learner must correctly answer five problems in a row. For help, a video explaining the topic and (usually) working through an example problem is available, as are hints. To motivate the learner, KA employs significant game mechanics features, such as points and badges.

Research on KA is scarce [2-4], with none in undergraduate physics education. From the same pilot project, Lindstrøm [5] reports that student compliance with KA was high (22 out of 24 used it and completed, on average, 22.3 of 28 prescribed topics), students' average performance on a diagnostic math test improved statistically significantly from pre-test $(42 \%, N=22)$ to post-test $(52 \%, N=21)$, and in the course evaluation, only two students responded negatively to the inclusion of $\mathrm{KA}$ in the course. In addition, KA was valuable for the instruc- tor by reducing class time spent revising math (resulting in time better spent in class), providing information about students' level of math competency (improving the tailoring of instruction to students), and helping identify at-risk students earlier (inviting them to remedial measures well before the end of the course).

\section{METHODOLOGY}

The context of this study is the fall 2014 Physics I course in pre-service science teacher education at the largest teacher education institution in Norway, Oslo and Akershus University College. The 24 students were in their fourth and final year of their Bachelor of Teacher Education degree. Physics I comprised seven 2h45min classes taught by C.L. in a course that employed Flipped Classroom, Just-in-Time Teaching and Peer Instruction [6]. KA was integrated into the pre-work, which also included reading the textbook, watching videos and doing a five-question online pre-test. The final examination carried $100 \%$ of the course grade, so students were encouraged to regularly complete their pre-work by allowing them to only hand in three out of the four problems in the compulsory course assignment if they achieved at least $50 \%$ of the pre-work marks available. Thus, using KA was not compulsory and compliance only carried a small external reward.

Two focus group interviews were conducted in spring 2015 by C.L. To solicit a diversity of responses, two groups of six students were invited: Group 1 (G1) comprised students who had used KA and had shown the highest improvement (avg. 20 percentage points) on a math diagnostic test [5], and Group 2 (G2) students who had used KA without showing much improvement (avg. 4 percentage points). All but one accepted the invitation. Students were informed that their responses would not affect their course performance in Physics II in any way, 
and were served pizza and soft drinks. A semi-structured interview guide with 11 questions was developed, which focused on students' impression of KA, motivations for using KA, the difficulty of the math in the physics course, and students' perceived contribution of KA to their understanding and performance in the course. The interviews were conducted in Norwegian, audio recorded (duration 47 minutes and 27 minutes respectively), transcribed, and analyzed by iterative thematic analysis with a grounded theory approach.

The analysis yielded one emergent theme for motivation and three themes for math in KA and physics. During the interviews, the students mentioned the motivating factors of both KA and the integration of KA in the course both before and after the specific question on motivation. The way math is included in a physics course plays an important role in motivation, so the next section is a self-contained analysis of these factors through the lens of Self-Determination Theory [7]. Motivation initiates and sustains the students' engagement with the subject matter and may also be a factor in the cognitive depth of that engagement [8]. In the subsequent section, we address the nature of this engagement, and relate it to the difficult problem highlighted by Redish and Kuo [1]. Unsurprisingly, there are no quick-fixes, but the analysis identifies potentially fruitful avenues for further research.

\section{STUDENTS' MOTIVATION FOR USING KA}

The most basic distinction within motivation is between intrinsic and extrinsic motivation [7]. Intrinsic motivation refers to doing something because it is inherently interesting or enjoyable, whereas extrinsic motivation is used to attain outcomes beyond those achieved from intrinsic motivation, employing rewards for desired behaviour ('carrot') or threat of punishment for misbehaviour ('stick').

Intrinsic motivation is revealed when activities are performed in the absence of separable consequences. In addition to satisfying the fundamental human needs for competence and autonomy, the activity must be inherently interesting to the individual. Factors facilitating competence building are optimal challenges, feedback promoting further exploration, and freedom from demeaning evaluations [7]. Extrinsic motivation is necessary when the activity is not inherently motivating, as is often the case in education. In this case, the reason for engagement is something other than personal interest. The perceived origin of this reason covers a broad continuum where the perceived locus of causality ranges from external to internal. The former is performed with resentment, resistance and disinterest, and the latter with an attitude of willingness reflecting an inner acceptance of the value or utility of the task [7].

The process of increasing the degree of one's internal locus of causality is known as internalization. Highly internalized extrinsic motivations are associated with greater engagement and higher quality learning, and are thus the most beneficial forms in education. Factors facilitating internalization are those that support autonomy, build perceived competence, and provide a sense of belonging to the person disseminating a goal (here the instructor) by feeling respected and cared for (called relatedness) [7].

All students freely admitted that the reward for completing the KA pre-work (one less problem on the compulsory assignment, referred to as "the carrot"), was an important extrinsic motivator for their use of KA, especially in the beginning. "I really like mathematics, but I do think that carrot plays a pretty big role in me actually bothering to spend an hour and a half, no, half an hour to an hour on it." "I was a bit surprised that-it's not a very big thing that carrot, but almost regardless of what it had been, I think, its better than nothing." The reward was deliberately small to support the students' feeling of autonomy. The key to student compliance with the prework throughout the semester, however, lay in their internalization of the extrinsic motivation. In both groups, students pointed out that although they started out being motivated by the carrot, working with KA made them realize that doing the pre-work was beneficial. "To begin with, the motivation was to avoid that [final] problem [in the compulsory assignment], and then you just end up spending an enormous amount of time on [KA] after each class. It's not just a duty, but you think it's okay and you learn something." They also experienced increasing competency through a non-demeaning feedback mechanism. "[Y]ou get good feedback all the time, and there's always good steady progress so you feel like you're always learning more math. So the motivation for me was at first that I would avoid the last problem in Physics, but after a while it was just fun to use KA." One student internalized their motivation to such a degree that it was almost indistinguishable from intrinsic motivation: "I didn't just do what we were supposed to; I went completely bananas. There was a Sunday when I just wanted to complete the whole of KA. So it is set up in such a way that it awakens your competitive instinct and you genuinely want to continue."

The students' only frustration with the carrot was when they felt unable to complete a topic through understanding and had to resort to less meaningful tactics, further emphasizing the importance of satisfying the need for competence and bringing the carrot into alignment with students' personal goals.

Finally, relatedness featured in students' experience of integration of KA in the course. They felt respected by the effort that went into picking appropriate pre-work to their level, and cared for by receiving emails when they were struggling with KA problems (offering early intervention measures for at-risk students). "I think that the teaching has been well organized, in that we have classroom teaching combined with pre-work to complete to get most out of class, and homework. And it is very structured, and you know what you have and haven't 
done. That's contributed to good progress in the course. And you also feel seen because [the instructor] sends an email if you've struggled with something. So really good follow-up. Very good set-up for the whole course, really."

\section{MATHEMATICS IN KA AND PHYSICS}

Despite significant variability among students regarding how difficult they found the math in the physics course, they all appreciated the contribution of KA to the course. There was unanimous agreement that KA helped with revising math the students had not engaged with for many years (often since high school) and filling in knowledge gaps (for which the videos were particularly helpful, although not all students were aware of these as they had not been explicitly introduced). As one student put it: "if there was something you knew already, then it was revision, so that was useful regardless. If there was something you didn't know, then you learned it."

'Mathematical mindset': Piaget's concept of assimilation is helpful to understand the students' description of learning in KA. Assimilation refers to development through refinement and expansion of existing mental schemata, as opposed to the more turbulent development that happens through accommodation in which the existing schemata cannot make sense of a new problem, information or situation and must be changed to deal with it [9].

The students described learning in KA as useful for getting them into a 'mathematical mindset,' a somewhat intangible expression used interchangeably with 'coming into the maths,' 'getting my head round the numbers,' 'getting maths under my skin,' and 'thinking mathematically.' "For me, I think KA helped with maintaining my math, with thinking mathematically. I don't feel that I learned so very much from using KA - a bit, of course - but it was more getting into a mathematical mindset." Note that the student does not think that they were learning when they were getting into a "mathematical mindset.' Using the framework above, we interpret the students' description of getting into a 'mathematical mindset' as a process of assimilation. The activity may not feel like learning to the student when compared to the more demanding process of accommodation. When viewed as assimilation, getting into a 'mathematical mindset' is a process where the math fits into the student's preexisting understanding, and involves organizing and effectivizing this understanding. However, by not classifying getting into a 'mathematical mindset' as learning, students might not be fully realizing the benefit of KA. Schoenfeld [10] points out that organizing one's understanding and streamlining information retrieval are important prerequisites to problem solving with math in real settings; thus, getting into a 'mathematical mindset' could be a vital but largely subconscious part of learning. We see exploring the nature and significance of the 'mathematical mindset' as an important avenue for fu- ture research.

Response to feedback: In the interview, five of the six G1 students mentioned issues that revolved around how the instant feedback and five-in-a-row requirement made them realize their lack of sufficiently deep understanding of certain topics: "Sometimes you got three in a row and think you've understood it, but, no, then you get one wrong. Then you have to spend time to understand it." In these cases, students would use the help (videos and hints) available in KA to understand the math. It is not clear exactly what the student means by understand, and we cannot assume that they imbue the word with the same meaning as experts do [1]; however, one student did make it clear that they did not consider purely procedural learning as understanding: "[I] had to learn a pattern [in the problems], because I'd never encountered it before, and the explanation in that video wasn't good enough for me to understand it."

The key point is that the G1 students were not satisfied with learning isolated procedures even when this was sufficient to complete a topic, displaying an inner drive for deeper cognitive engagement with the material [8]. This attitude was not expressed by the G2 students. It is well documented that students that actively monitor their comprehension perform better academically, and deeper cognitive engagement can lead to better performance on tests that measure synthesis, analysis and deep-level understanding [8]. These results mirror the performance on the math test of G1 and G2. The G2 students also expressed a lack of connection between the KA work and the math test, indicating that their learning was not as synthesized as the G1 students.

We see fruitful directions for future research probing what students mean by understanding in the context of working with math topics in KA and exploring the cognitive processes of students representative of the two different groups while they work on problems in topics and receive feedback, e.g., in think-aloud interviews. This could subsequently lead to investigating what type of interventions are most effective in encouraging and training students who do not of their own volition search for deeper understanding, and whether such interventions have the power to reduce or even eliminate the gap between these two groups of students.

Mathematics in physics: All KA topics were chosen because they were considered relevant preparation for class. However, this was rarely pointed out, with the result that both groups commented that they did not always see the application of the KA topics to class content or activities. This was more problematic for G2 than G1, due to a lower level of cognitive proactivity in response to realizing the lack of expected connections.

All G1 students explicitly expressed that despite the occasional lack of apparent relevance, KA was still valuable for getting into the 'mathematical mindset', which helped them better follow what happened in class. This reduced their need for the instructor explaining as much math on the board and enabled them to explain math to 
each other during group work. "You notice very quickly when [fellow students] haven't also done the math prework. You notice that they haven't gotten into it. It might be that they haven't understood it, but that's another matter." There were indications that the initial exposure to math allowed students to gain deeper understanding in class. One student described the contribution to solving a difficult problem: "Refreshing the math helped me understand, but it was still difficult to understand." It seems that the KA pre-work enabled this student the opportunity to problem solve - the problem was difficult but they managed it because they had refreshed their math. Such enabling is according to Schoenfeld a characteristic of mathematical expertise [10].

The G2 students, in contrast, did not appear to search for connections in the same way as the G1 students, and continued to express a desire for connections between KA topics and class material to be made explicit. We see this as empirical evidence for the analytical findings of Redish and Kuo [1]: "Even if students have learned the relevant mathematical tools in their math courses, they still need to learn a component of physics expertise not present in math class - tying those formal mathematical tools to physical meaning" (p. 583). This penchant for searching for connections among the G1 students that we do not see in the G2 students could represent a crucial distinction between the success of the two groups.

Fruitful research avenues include investigating what cognitive actions - if any - students choose to take when they realize a lack of expected connection between math and physics (such as in the context of using KA for prework), causes of action or non-action (e.g., relating to self-efficacy for finding such connections: if students do not believe they are able to find these connections themselves, they are less likely to search for them), and which interventions are most effective in helping students forge such connections.

For G1, not only did math support the physics, but physics supported the math. Schoenfeld's [10] elaboration on the metaphor of math as a kitchen allows parallels between the role of recipes in cooking and procedures in math: "having basic procedures down cold ... is essential in mathematics and in the kitchen ... I did not spend the first $n$ years of my culinary life ... practicing boiling, poaching, sautéing ... before I was allowed to make full recipes. Basic skills were learned, sometimes with drill, in the context of meaningful work" (p. 313). Physics is an example of such meaningful work in which we use mathematical procedures in a context rich with physical meaning. "I think that the math in physics has an advantage, because the numbers in the calculations aren't so abstract, really, because you have that weight of something - a real thing. It's not just a quadratic equation, 'get going'. Here you have to figure out what you're dealing with as you go." In the words of Redish and Kuo [1], "we not only use math in doing physics, we use physics in doing math" (p. 563, italics in original).

\section{CONCLUSION}

Returning to the difficult problem [1] of "how to effectively include math in physics in a way that reaches most students" (p. 561), we see that the high levels of student compliance reported in [5] are explained by the features of KA and the integration of $\mathrm{KA}$ in the course leading to an internalization of the extrinsic motivating factors. These features are an important first step for the math to reach most students. An analysis of the nature of the students' engagement reveals the importance of a fuzzy and largely subconscious knowledge network in the 'mathematical mindset' and a natural preponderance to identify connections between math and physics among only some students, who were also the only ones to appear to engage in deeper processing of the feedback provided by KA. We see integrating KA into physics as a practical and productive test-bed for further exploring the nature of student predilection to search for understanding, and the meaning and role of 'mathematical mindset'.

\section{ACKNOWLEDGEMENTS}

We would like to thank MatRIC for project funding, Hilde Midtgård Stephansen and Ina Camilla Lauvli Engan for transcribing the interviews, Kristian Vestli for his contribution to the project team, and Yvette Solomon, Tor Ole Odden, Michelle Rigozzi and Morten Misfeldt for valuable feedback on the paper.
[1] E. F. Redish and E. Kuo. Sci. \& Educ. 24, 561 (2015).

[2] R. Murphy, L. Gallagher, A. Krumm, J. Mislevy and A. Hafter. Research on the Use of Khan Academy in Schools (SRI Education, Menlo Park, CA, 2014).

[3] S. G. Wilson, Teaching of Psychology 40, 193 (2013).

[4] J. Gray, C. Lindstrøm, and K. Vestli. Proc. CERME10 (Dublin, Ireland, 2017).

[5] C. Lindstrøm. Proc. ASEE (ASEE press, Seattle, WA, 2015), p. 9093.
[6] C. Lindstrøm and J. Schell. Actas del VI Simposio Las Sociedades ante el Reto Digital (2013), p. 7.

[7] R. M. Ryan and E. L. Deci, Contemp. Educ. Psych. 25, 54 (2000).

[8] J. A. Fredricks, P. C. Blumenfeld and A. H. Paris, Review of Educational Research 77, 59 (2004).

[9] J. Piaget, Construction of reality in the child (Basic Books, New York, 1954).

[10] A. Schoenfeld, in Thinking Practices in Mathematics and Science Learning edited by J. G. Greeno and S. V. Goldman (Routledge, New York and London, 1998). 